\section{Dysregulated fibulin-5 expression and elastogenesis in COPD lungs: pyromaniac or fire fighter?}

\author{
Guy Brusselle $e^{1,2}$
}

COPD is characterised functionally by a persistent airflow limitation, which is not fully reversible and is usually progressive. The pathological hallmarks of COPD are, on the one hand chronic inflammation and remodelling of the small airways (ie, bronchiolitis) and, on the other hand enlargement of alveolar spaces due to destruction of alveolar lung tissue (ie, emphysema). ${ }^{1}$ Fibrosis of the small airways and loss of elastic recoil due to emphysematous destruction contribute to chronic airflow limitation in COPD. Elastic fibres, principal components of the extracellular matrix (ECM) of alveolar septa, provide mechanical elasticity to the lungs and interconnect the lung parenchyma with small airways via alveolar attachments. An imbalance between proteases (such as neutrophil elastase) and antiproteases (such as $\alpha 1$-antitrypsin, the main inhibitor of neutrophil elastase), leading to a net increase in proteolytic activity, has been implicated in the pathophysiology of COPD. ${ }^{2}$ However, two opposing processes are supposed to drive the pathogenesis of small airway fibrosis and emphysema: the thickening of the small airway walls results from an exaggerated repair process leading to a marked increase in deposition of ECM, whereas the emphysematous destruction of the lung tissue is indicative of an insufficiënt production of ECM and, thus, a defective repair. Since small airway fibrosis and emphysema occur in close proximity to each other in COPD, the underlying mechanisms of their pathogenesis remain enigmatic.

C-A Brandsma and collaborators add an important piece to the puzzle. ${ }^{3}$ Thanks to an international collaboration between three academic centres (Groningen, Quebec and Vancouver, as partners within the Lung eQTL Consortium), they demonstrate-

\footnotetext{
${ }^{1}$ Department of Respiratory Medicine, Ghent University Hospital, Ghent, Belgium; '2Departments of Epidemiology and Respiratory Medicine, Erasmus Medical Center, Rotterdam, The Netherlands

Correspondence to Dr Guy Brusselle, Department of Respiratory Medicine, Ghent University Hospital, De Pintelaan 185, Ghent B-9000, Belgium; guy.brusselle@ugent.be
}

using genome-wide gene expression profiling of a large number of lung tissue samples -that 112 genes are significantly upregulated in patients with COPD compared with non-COPD controls, and that 61 genes are downregulated (including vascular endothelial growth factor $\alpha$ (VEGFA) and periostin (POSTN)). Among the most upregulated genes, they discovered four genes which are involved in ECM organisation and elastogenesis: fibulin-5 (FBLN5), elastin $(E L N)$, latent transforming growth factor $\beta$ (TGF $\beta$ )-binding protein 2 (LTBP2) and microfibrillar-associated protein 4 (MFAP4). Importantly, they validated the array gene expression findings using quantitative PCR (qPCR) on lung tissue samples from the Groningen cohort, confirming increased messenger RNA (mRNA) expression of fibulin-5, ELN and MFAP4 in COPD. Moreover, they found significant negative correlations between the expression of these three elastogenesis genes and disease severity $\left(\mathrm{FEV}_{1}\right)$. Since pulmonary fibroblasts are the main source of ECM proteins in the lung, and since TGF $\beta$ is a key driver of remodelling processes, the authors investigated the FBLN5 gene expression levels in lung fibroblasts in vitro, and demonstrated a significant increase in fibulin-5 mRNA expression after treatment with TGF $\beta$. Finally, the investigators examined the protein expression of fibulin-5 in the lung by immunohistochemistry and western blot. The histochemical analysis revealed clear colocalisation of fibulin-5, LTBP2 and MFAP4 with elastic fibres in the lung, particularly in alveolar walls and around the airways. Interestingly, western blot analysis demonstrated that total fibulin-5 protein levels in lung tissue were significantly higher in COPD subjects than in controls, and also highlighted the presence of a cleaved, non-functional form of fibulin-5 in COPD. Since serine proteases, such as neutrophil elastase are vastly increased in the lungs of patients with COPD and can cleave fibulin-5 into a nonfunctional protein, increased levels of cleaved non-functional fibulin-5 are unable to contribute to the appropriate assembly of elastic fibres and tissue repair in COPD. Thereby, the attempt to enhance elastogenesis in COPD could be ineffective in counterbalancing the increased proteolytic breakdown of the lung ECM. Of course, this hypothesis should be tested appropriately.

The lung gene expression study by Brandsma et al suggests that fibulin-5 might be a key player in the pathogenesis of COPD. Fibulin-5 plays a crucial role in the assembly of elastic fibres, which are composed of microfibril proteins (such as fibrillins and latent TGF- $\beta$ binding proteins (LTBP)) and polymerised elastin. Fibulin-5 is abundantly expressed in the lung during fetal development and is reactivated in adulthood upon tissue injury. Interestingly, FBLN5 knockout mice develop severe emphysema and other manifestations of a general elastinopathy, including a loose skin and vascular abnormalities. ${ }^{4} 5$ In humans, mutations in the FBLN5 gene can cause autosomal recessive cutis laxa (loose skin) syndrome, a hereditary connective tissue disorder characterised by fragmentation of elastic fibres and associated with severe emphysema. ${ }^{6}$ Recently, another member of the fibulin family of ECM glycoproteins-fibulin-3has been implicated in diverse respiratory disorders. A large genome-wide association (GWA) study has associated single nucleotide poylmorphisms in the EFEMP1 gene, encoding fibulin-3, with forced vital capacity (FVC). ${ }^{7}$ Moreover, fibulin-3 protein levels-measured in plasma or pleural fluid with an ELISAappears to be an accurate diagnostic biomarker for patients with mesothelioma. ${ }^{8}$

The paper by Brandsma et $a l^{3}$ not only provides intriguing insights in the pathogenesis of COPD, it also is exemplary for gene expression studies because the results of the large genome-wide mRNA expression profiling in lung tissue are based on an international collaboration between three academic centres, which performed separate analyses per cohort followed by a meta-analysis. Collaborative research within large consortia has become standard state-of-the art methodology in genetic studies such as GWA studies, but has, until now, only rarely been applied to expression studies in the respiratory field. The assets of large-scale collaborations in GWA studies, encompassing replication of significant associations in independent cohorts and meta-analysis of results obtained in separate smaller cohorts are obvious, and have vastly improved the external validity of the results of genetic studies. ${ }^{79}$ We welcome a similar collaborative approach by the Lung eQTL Consortium for lung gene expression studies. ${ }^{3}$

What are the next steps? First, since the expression studies were performed on 


\section{Editorial}

whole lung tissue resections, it would be worthwhile to pinpoint the exact localisation of the increased expression of fibulin-5 in the diseased lung in COPD (alveolar compartment vs small airways) via laser capture microdissection and/or confocal microscopy. Second, and more importantly, we need to address the functional role of fibulin-5 in the pathogenesis of COPD by translational and preclinical research in animal models in vivo. Is the vastly increased mRNA and protein expression of fibulin-5 in the lung causing the fibrosis of small airways in COPD, and thus detrimental (ie, pyromaniac)? Or is the increased elastogenesis gene expression in the lungs of patients with COPD an attempt to repair the large-scale damage and destruction of the alveolar walls, and thus beneficial (ie, fire fighter)? Last, can fibulin-5 protein be detected in blood and/ or sputum, and could it be used as a diagnostic or prognostic biomarker in COPD? Independent of the answers on these questions, we thank the Lung eQTL
Consortium for putting the fibulin family on the COPD research agenda.

Competing interests None.

Provenance and peer review Not commissioned; internally peer reviewed.

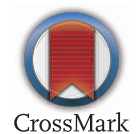

To cite Brusselle G. Thorax 2015;70:1-2.

Published Online First 7 August 2014

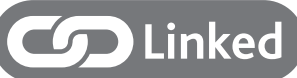

http://dx.doi.org/10.1136/thoraxjnl-2014-205091

Thorax 2015;70:1-2.

doi:10.1136/thoraxjnl-2014-205887

\section{REFERENCES}

1 Hogg PJC. Pathophysiology of airflow limitation in chronic obstructive pulmonary disease. Lancet 2004;364:709-21.
2 Brusselle GG, Joos GF, Bracke KR. New insights into the immunology of chronic obstructive pulmonary disease. Lancet 2011;378:1015-26.

3 Brandsma C-A, van den Berge M, Postma DS, et al. A large lung gene expression study identifying fibulin-5 as a novel player in tissue repair in COPD. Thorax 2015;70:21-32.

4 Yanagisawa $H$, Davis EC, Starcher BC, et al. Fibulin-5 is an elastin-binding protein essential for elastic fibre development in vivo. Nature 2002;415: 168-71.

5 Nakamura T, Lozano PR, Ikeda Y, et al. Fibulin-5/ DANCE is essential for elastogenesis in vivo. Nature 2002;415:171-5

6 Callewaert B, Su C-T, Van Damme T, et al. Comprehensive clinical and molecular analysis of 12 families with type 1 recessive cutis laxa. Hum Mutat 2013;34:111-21.

7 Loth DW, Artigas MS, Gharib SA, et al. Genome-wide association analysis identifies six new loci associated with forced vital capacity. Nat Genet 2014;46:669-77.

8 Pass HI, Levin SM, Harbut MR, et al. Fibulin-3 as a blood and effusion biomarker for pleural mesothelioma. N Engl J Med 2012;367:1417-27.

9 Hancock DB, Eijgelsheim M, Wilk JB, et al. Meta-analyses of genome-wide association studies identify multiple loci associated with pulmonary function. Nat Genet 2010;42:45-52. 